\title{
RUNA SONCCO: MANUEL NÚÑEZ BUTRON Y SU PROYECTO DE EDUCACIÓN SANITARIA
}

\begin{abstract}
C. Hugo Arroyo-Hernández $1,2, a$
RESUMEN

Runa soncco palabra quechua que significa "corazón de indio" es el título de una revista publicada del año 1935 al 1948, cuyos artículos reflejan el deseo por integrar la cultura andina con la salud pública occidental, desde el mismo campo de acción. Esta publicación logró situarse como una herramienta fundamental en la difusión del mensaje sanitario y de educación del movimiento indigenista liderado por el médico Manuel Núñez Butrón. Constituye una de las primeras experiencias en atención preventivo-promocional en el Perú y el mundo, anticipándose a lo que cuatro décadas más tarde se denominaría "atención primaria de la salud".
\end{abstract}

Palabras clave: Salud pública; Educación sanitaria; Atención primaria de salud; Perú (fuente: DeCS BIREME).

\section{RUNA SONCCO: MANUEL NUÑEZ BUTRON Y SU PROYECTO DE EDUCACIÓN SANITARIA}

\section{ABSTRACT}

Runa soncco, a Quechua term for "Indian heart", is the title of a magazine published between 1935 and 1948, which articles reveal the intention to integrate the Andean culture with Western public health on-site. It also turned into a key tool for the dissemination of the sanitation and education message and the indigenous movement led by Dr. Manuel Núñez Butrón, one of the first experiences in promotional preventive care in Peru and in the world - a pioneer of what four decades later would be called "primary health care".

Key words: Public health; Education, medical; Primary health care; Peru (source: MeSH NLM).

\section{RIJCHARISMO, UN ANTECESOR DE LA ATENCION PRIMARIA EN SALUD}

En el año 1933, el médico peruano Manuel Núñez Butrón asume la iniciativa de aplicar una verdadera labor social, crear un sistema de atención sanitaria orientado a las poblaciones indígenas y campesinas de Puno, lo cual generó un movimiento regional al que denominó rijcharismo, término que deriva del quechua rijchary (despierta) y que sentó sus bases en la salud, la educación y el trabajo, y que se convirtió en una de las primeras experiencias de atención preventivopromocional en el Perú y el mundo, anticipándose a lo que cuatro décadas más tarde se llamaría "atención primaria de la salud" ${ }^{(1,2)}$.

Es el mismo Núñez Butrón quien organiza en brigadas sanitarias, o richarys, a curanderos, herbolarios, exsoldados y demás pobladores de la zona, quienes viajaban de una comunidad a otra atravesando largas distancias a pie o en moto, para enseñar a los indígenas (Figura 1) el cuidado de su propia salud y la práctica de la higiene. Para ello, enseñaban a fabricar jabones, desinfectar habitaciones, así como las causas de las enfermedades y las ventajas de la vacunación, utilizando canciones populares con letras que promovían la alfabetización y la higiene, o presentando espectáculos de teatro al aire libre en lenguaje nativo; además de realizar actividades asistenciales que podían ir desde una exodoncia, hasta la atención de un parto ${ }^{(3)}$.

\section{RUNA SONCCO, LA PUBLICACIÓN COMO HERRAMIENTA DE EDUCACIÓN DEL RIJCHARISMO}

Una de las herramientas fundamentales en la obra de Núñez Butrón fue la difusión de su mensaje sanitario

\footnotetext{
Oficina General de Información y Sistemas, Instituto Nacional de Salud. Lima, Perú.

CRONICAS Centro de Excelencia en Enfermedades Crónicas, Universidad Peruana Cayetano Heredia. Lima, Perú.

a Médico cirujano.

Recibido: 22-05-13 Aprobado: 05-06-13
}

Citar como: Arroyo-Hernández CH. Runa soncco: Manuel Nuñez Butron y su proyecto de educación sanitaria. Rev Peru Med Exp Salud Publica. 2013;30(2):336-9. 




Figura 1. Rijcharismo. En la imagen se presenta una campaña de salud en el altiplano de Puno. Se aprecia, un brigadista sanitario rural o rijchary -identificado con un brazalete y una cruz,personas de ambos sexos y la participación de diferentes actores sociales como el médico (Manuel Núñez Butrón), un oficial del ejército y probablemente autoridades locales. Asimismo, la lectura de documentos educativos a la población

Fuente: Dr. Manuel Núñez Butrón, pionero de la atención primaria en el mundo, disponible en: http://alarv3.wix.com/manuelnunezbutron

y de educación a través de la revista Runa Soncco, palabra quechua que según uno de sus colaboradores va más allá de su traducción literal "corazón de indio", debiéndose entender como "hombre que tiene mucho amor al indio" (4).

Se publicaron un total de diez números (cinco en 1935, dos en 1937, y uno en 1945,1946 y 1948). El contenido de cada número de Runa Soncco estaba adaptado a la mentalidad y necesidades de las comunidades indígenas de Puno, por lo que se publicaron artículos tanto en castellano como en quechua y aymara. Se puede encontrar, además, en cada número de la revista un espacio para la vida social de los indígenas, con noticias sobre servinacuy, matrimonio, defunciones o nacimientos, las cuales están acompañadas de imágenes de las actividades realizadas por los richarys, así como de expresiones culturales indigenistas, representadas por el xilografista y artista Flores $\mathrm{F}^{(5)}$ (Figura 2).

Una revista de esta naturaleza es imposible de comprender sin antes tener presente el contexto del movimiento indigenista que se desarrolló en el Perú de aquella época. En la sierra de Puno, este se expresó como un indigenismo médico el cual señalaba que el problema que afrontaba el indígena era básicamente "un problema sanitario" (6). Es así que en Runa Soncco se puede encontrar un evidente deseo por integrar la cultura andina con la salud occidental, mediante la reivindicación de la raza indígena y de su cultura. A lo largo de sus páginas la revista combina, temas indigenistas con el mensaje sanitario, difundiendo sugestivos lemas, como Desconocer su raza indica ignorancia o Qué rica es la comida cuando las manos están bien lavadas.

Por aquellos años el tifus y la viruela eran enfermedades endémicas en Puno y en el resto de regiones de la sierra central y sur del Perú ${ }^{(7)}$. Estos padecimientos eran aceptados por los indígenas como algo cotidiano e inevitable, tal como lo refleja, un artículo en Runa Soncco en el que se menciona que: La viruela y el tifus son dos enfermedades que cuando visitan una casa se les recibe afablemente y cuando se van, se les despide con los honores que merecen. La revista, criticaba abiertamente los comportamientos y malos hábitos que favorecían la diseminación del tifus, como no cortar el pelo a los niños, colocar a los niños en la cama del enfermo de viruela, o la desconfianza de la población indígena hacia la vacunación. En Runa Soncco también se describió sesiones de debate entre los rijcharys y los campesinos sobre el origen mágico o científico de la viruela. Durante la primera mitad de 1937, los rijcharys realizaron mil vacunaciones y cortaron el pelo a poco más de seiscientas personas ${ }^{(3)}$.

La revista incluía comunicaciones tales como adivinanzas, poemas, y correspondencias al director. Es interesante encontrar en un número, un artículo, que asemeja un reporte de caso, titulado "Historia clínica autóctona de la enfermedad de doña Asunta Pilco". La descripción del interrogatorio, antecedentes paternos, personales y el relato de la enfermedad actual impresionan por el contenido de datos etiológicos y descripciones onomatopéyicas de la enfermedad, reflejando lo complejo de la concepción e interpretación del proceso de salud y enfermedad por las mujeres y hombres del ande ${ }^{(8)}$. Otro ejemplo, igual de rico en sus descripciones es la correspondencia al director firmada el 4 de junio de 1935 por el sanitario rijchary Martin Apaza. Textualmente se indica: Tengo una noticia para decil a Ud. en cuanto de una persona de que esta enfermando muy grave y tan triste mi doctor, esta enfermedad parece que, sopla de frio y calor, si siente todo el cuerpo pero está picando como una aguja todo el cuerpo de su pie y de su variga y de sus manos de todo dispuis si sale sodor frio y no se puede aguantar, ya está tres meses. Tambien su varega esta inchadu, un poco no tanto regular, pero nosotros dicemos cólera erretación, no se mi doctor" (9).

Uno de los aspectos más importantes del rijcharismo, y de Manuel Nuñez Butron, es el carácter inclusivo, que se propuso para difundir los contenidos de Runa Soncco. Considerando que muchos indígenas eran analfabetos, la revista era leída en voz alta por los mismos richarys, quienes se encargaron además de difundir otras publicaciones como diarios o folletos que 


\section{RUNA SONCCO}
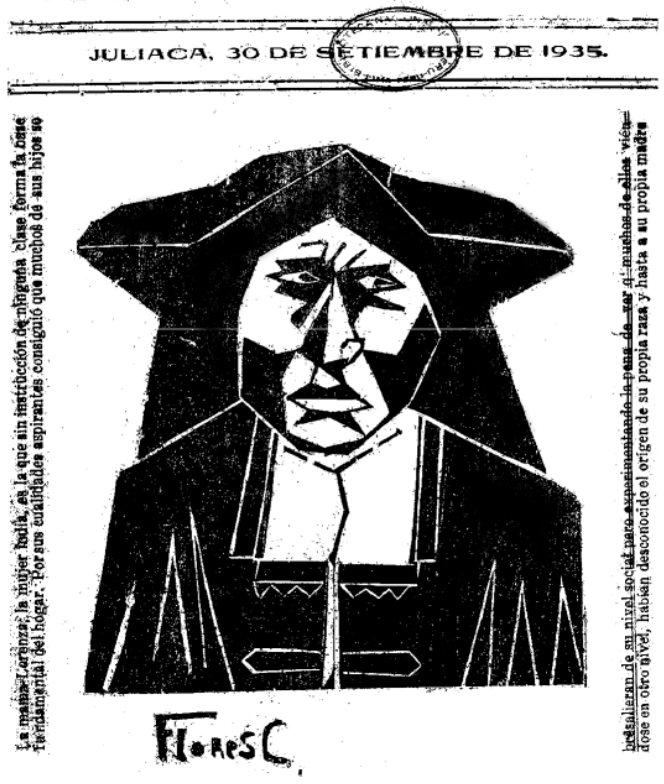

Figura 2. Carátula del número cinco de Runa Soncco, la descripción alrededor de la imagen rescata el rol de la mujer indígena, en su rol de madre, como base fundamental del hogar (fuente: Hemeroteca de la Biblioteca Nacional del Perú)

remitía la dirección de salubridad, en una especie de biblioteca ambulante que recibía suscripciones gratuitas o por canje de otras publicaciones.

El principal financiamiento de la revista era posible gracias al propio Núñez Butrón, a subscripciones y la cooperación de seguidores. La revista llegó a tener difusión internacional siendo reconocida por higienistas de Chile y Argentina. Asimismo la United Press, reproducía sus artículos en catorce puntos del extranjero ${ }^{(10)}$, una de las carátulas de la revista lleva el sello de "\$ 0,20", señalando, probablemente, el valor de la revista en algún país del extranjero. Sin embargo, su falta de continuidad durante los últimos años se pudo deber a necesidades económicas, tal como se relata en uno de sus artículos: Runa Soncco, se vende cuando quieran pagar porque generalmente se regala a quienes consideramos que lo necesitan o reconocemos que tiene esta misma tendencia educadora; pero como estamos en vísperas de nadar, ya que el agua nos está llegando al cuello, suplicamos nos ayuden quienes consideren correcta esta campaña en el mundo de los piojos, del alcohol y la pleitomania ${ }^{(11)}$.

Sin embargo, esta concepción de revista no fue del completo entendimiento de sus lectores. En un artículo titulado "El vocero de los indios y para los indios", publicado en el tercer número de Runa Soncco ${ }^{(12)}$, se relata: Por qué hay tanto oposición para que los indios tengamos un vocero, cuando la lectura de revistas nos dice que en Paris, Londres, Berlín, los carpinteros, los sastres, choferes, cocineros, tienen sus periódicos. También nos dicen que en Nueva York hasta los ciegos disponen de discos grabados con las noticias del día; en otra parte del mismo artículo se dice: Los indios tenemos nuestro periódico a fuerza de tanto sufrimiento. Los que no son indios casi siempre le preguntan a nuestro director: ¿Por qué a Coaquira lo pone usted en sociales y no me pone usted a mí? responde nuestro director "Sencillamente porque usted no es indio".

La mejor descripción sobre lo que significó esta publicación y el Rijcharismo, se encuentra en su último número publicado el 24 de junio de 1948: Runa Soncco es el periódico de los indios y para los indios. Allí está parte de todo lo que se ha hecho. En la mayor parte de Runa Soncco nosotros éramos simples traductores y al final siempre hemos visto que llegaron a escribir. Tuvimos la suerte de hacer escribir y escribiendo el comportamiento altipampino creíamos que el problema se había resuelto, Runa Soncco era el pan espiritual del campo y quien tuvo la suerte de que llegara a sus manos, en la actualidad es un documento familiar. Por Runa Soncco tenía más deseos de aprender a leer. Por Runa Soncco les despertaba el apetito de vivir mejor ${ }^{(8)}$.

\section{REFLEXIONES FINALES}

En la actualidad solo cinco números se encuentran disponibles en la Biblioteca Nacional del Perú, es probable que la obra de Núñez Butrón no sea del todo conocida por muchos médicos; en particular entre aquellos de generaciones más jóvenes ${ }^{(13)}$, por lo que la importancia de Runa Soncco debe volver a ser valorada y sus artículos socializados por instituciones académicas en ciencias de la salud quienes pueden encontrar en esta revista una original e interesante fuente de referencia de lo que significa evitar para no curar, con inclusión y participación de la comunidad. 


\section{REFERENCIAS BIBLIOGRÁFICAS}

1. Neyra J. "Héroes de la Salud Pública en el Perú" Manuel Núñez Butrón (1900-1952). Rev Peru Med Exp Salud Publica. 2005;22(2):148-9.

2. Knipper M. Más allá de lo indígena: salud e interculturalidad a nivel global. Rev Peru Med Exp Salud Publica. 2010;27(1):94-101.

3. Cueto M. El regreso de las epidemias: salud y sociedad en el Perú del siglo XX. Lima: Instituto de Estudios Peruanos; 1997.

4. Mollepasa A. Lo que quiere decir runa soncco. Runa Soncco. 1937;6:2.

5. Frisancho-Pineda D. Manuel Nuñez Butron y el «Rijcharismo». Act Med Peru 2001;8(2).
6. Leonardini N. El grabado en el Perú republicano: diccionario histórico. Lima: Universidad Nacional Mayor de San Marcos; 2003.

7. Frisancho D. Las dos últimas epidemias de viruela en el Perú. Rev Peru Epidemiol. 1996;9(1):54-7.

8. Runa Soncco. Runa Soncco. 1935;3:13.

9. Apaza M. Una comunicación literal de «Chacas». Runa Soncco. 1935;4.

10. Colegio Médico del Perú, Comité de Educación Médica Contínua. Dr. Manuel Núñez Butrón. Pionero de la Atención Primaria en el Mundo [Internet]. Lima: CMP; c2011 [citado 19 de mayo de 2013]. Disponible en: http:// alarv3.wix.com/manuelnunezbutron
11. Pobre Runa Soncco. Runa Soncco. 1946;9.

12. El vocero de los Indios y para los Indios. Runa Soncco. 1948;3:2.

13. Pamo-Reyna O. Hacia un nuevo Rijcharismo. Bol Soc Peru Med Interna. 1994;7(4):139-40.

Correspondencia: Hugo Arroyo Hernández Dirección: Instituto Nacional de Salud, Cápac Yupanqui 1400, Lima 11, Perú Teléfono: 964579990

Correo electrónico: hugoarroyo2007@gmail. com

\section{Consulte las ediciones anteriores de la Revista Peruana de Medicina Experimental y Salud Pública en} www.scielosp.org

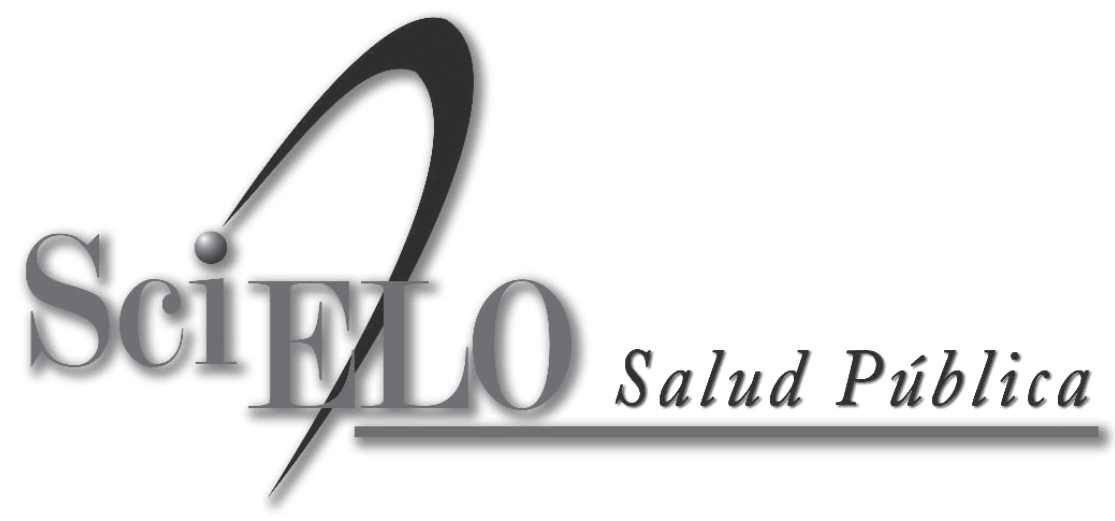

